\title{
Effectiveness of Dry Needling in Bothersome Chronic Tinnitus in Patients with Myofascial Trigger Points
}

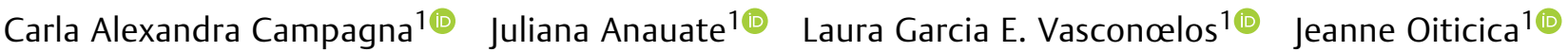 \\ 1 Otolaryngology Department, Faculdade de Medicina da \\ Universidade de São Paulo, São Paulo, SP, Brazil \\ Address for correspondence Jeanne Oiticica, PhD, University of Sao \\ Paulo-Otorhinolaryngology, Alameda Dos Jurupis 657 CJ 72 \\ Indianopolis, Sao Paulo, Sao Paulo 04088002, Brazil \\ Int Arch Otorhinolaryngol 2022;26(2):e233-e242. \\ (e-mail: jeanneramalho@uol.com.br).
}
Abstract
Keywords
- tinnitus
- physiotherapy
- somatosensorial tinnitus
- dry needling
- trigger points
- placebo-controlled

Introduction Therapeutic dry needling (DN) is effective in reducing the discomfort of chronic somatosensory tinnitus in patients with myofascial trigger points (MTP)s.

Objective To evaluate the efficacy of DN in chronic somatosensory tinnitus discomfort in patients with MTP.

Methods Placebo-controlled paired trial that included 16 patients with a diagnosis of somatosensory chronic tinnitus and with the presence of at least one active or latent MTP. Treatment was performed in two phases: (1) four sessions (one session per week for four consecutive weeks) of placebo DN and (2) four sessions of therapeutic DN with a gap (washout) of 15 days between these phases.

Results The Tinnitus Handicap Inventory (THI) variable and its emotional domain had a statistically significant reduction in therapeutic DN when compared with placebo DN ( $p=0.024$ and $p=0.011$, respectively).

The tinnitus visual analogic scale (VAS) signaled a reduction in tinnitus discomfort when compared with moments before and after therapeutic DN $(p<0.05)$.

Conclusion The therapeutic DN technique for MTP in patients with chronic tinnitus of somatosensory origin proved effective in reducing symptom discomfort, as measured by the THI (total score) and its emotional domain when compared with placebo DN.

\section{Introduction}

Tinnitus can be defined as sound perception in the head or in the ear in the absence of an external environmental sound source. ${ }^{1}$ Epidemiological data show that the prevalence of tinnitus in the city of São Paulo is of $22 \%^{2}$

Tinnitus is a symptom that can be triggered by several causes and, in general, two or three etiological factors can be identified in the same patient. Among these factors, auditory and somatosensory system disorders stand out. The somatosensory subtype has as its main characteristic modulation in tinnitus awareness (intensity, frequency, location) from forced contraction maneuvers of the head and/or neck muscles. ${ }^{3}$ According to this Levine ${ }^{3}$, this subtype of subjective tinnitus can be present in about $68 \%$ of patients.

A possible explanation for this phenomenon might lie in the existing connections between auditory and somatosensory pathways. This has previously been shown in the literature through a study in which an auditory stimulus (the sound of rubbing hands) managed to trigger a tactile sensation (dryness sensation of the palms). ${ }^{4}$ received

October 13, 2020

accepted

DOI https://doi.org/

10.1055/s-0041-1730429.

ISSN 1809-9777. 
Experimental studies have shown that in the face of an installed hearing loss, changes in (pre-existing) neural connections between the trigeminal and dorsal spine pathways occur (gracile and cuneiform nuclei) over the dorsal cochlear nucleus in the brainstem. ${ }^{5}$ These changes involve the amount and expression of glutamate receptors and of auditory and proprioceptive pathways on this nucleus. This rearrangement of neural connections culminates in the increase of somatosensory influence on the dorsal cochlear nucleus. This evidence supports, once again, the relevance of cross modal neural plasticity (reorganization ability between two or more sensory modalities) for the generation of tinnitus. ${ }^{5}$

Somatosensory tinnitus may be related to the existence of myofascial trigger points (MTPs) in the head musculature and/or ${ }^{7}$ in the neck and/or shoulder girdle, to temporomandibular joint (TMJ) disorders, and/or to craniocervical proprioceptive disorders. ${ }^{6,7}$

The association between tinnitus and MTPs has already been widely discussed in the literature. ${ }^{8-13}$

Myofascial trigger points are hyper-irritable areas located in a tense band of the skeletal muscle. They can be active or latent, depending on their clinical characteristics. An active MTP causes spontaneous resting pain, and its palpation generates a reported pain pattern, similar to the pain complaint of the patient. Latent MTPs may not cause spontaneous pain, and may only appear in the face of the local stimulus; however, they can have repercussions, which may include decrease of range of motion (ROM) as well as muscle weakness. $^{8}$

Clinical complaints related to MTPs (pain, limitation of ROM, paresthesia, and weakness) occur by the shortening of muscle fibers and pressure on adjacent nerves and body tissues. $^{14}$

Treatment modalities already described for MTPs include: (1) antidepressants, neuroleptics, non-hormonal anti-inflammatory drugs, ${ }^{15-17}$ (2) acupuncture, (3) ultrasonography therapeutic application (longitudinal and compressional sound waves that travel through biological tissue and induces mechanical vibration as well as displacement of molecules), (4) diathermy, (5) transcutaneous electrical neural stimulation (TENS), (6) cold spray (cryotherapy resource used as a local anesthetic in physical therapy), (7) several stretching techniques, (8) dry needling (DN), and (9) local injections with anesthetics and/or steroids. ${ }^{18,19}$ The clinical efficacy of these therapies is yet to be defined, since studies comparing pre-and post-treatment outcomes with control and placebo groups are scarce.

Dry needling works as a mechanical stimulus and physical agent that treats MTPs in the affected muscle tissue by inserting a long thin needle. This results in a decrease of muscle pain and stiffness, in an improvement in the ROM, and in a balance in muscle strength and function. ${ }^{20-22}$ The technique does not require injection of any drug and may result in local muscle spasm. Triggering, etiological and perpetuating factors should be corrected to prevent relapse.

Although DN has been described for some time as a therapeutic strategy in the treatment of MTPs, ${ }^{23}$ it has never been tested in patients with chronic tinnitus of somatosen- sory origin. Our hypothesis is that treating MTPs through DN is effective in reducing tinnitus discomfort.

\section{Methods}

The present clinical trial was placebo-controlled and crossover (ClinicalTrials.gov Identifier: NCT03295890). It was previously submitted to the Research Ethics Committee of our institution and was approved under the Process Opinion Number 1.784.310, CAAE: 60675516.4.0000.0068 of October $20^{\text {th }}, 2016$ ). All subjects of the present trial have authorized and signed the free and informed consent form (FICF).

\section{Placebo and Therapeutic Groups}

After a previous pilot project, 16 patients were selected.

The selection criteria included: (1) age $>18$ years old, (2) both genders, (3) constant tinnitus, (4) unilateral or bilateral, (5) for at least 6 months, (6) presence of at least 1 MTP (active or latent) in the head, neck and/or shoulder girdle.

The criterion for the diagnosis of somatosensory tinnitus included the presence of tinnitus (intensity, frequency, location) modulation (exacerbation, onset, attenuation) during passive palpation of the head and neck muscles by one of the researchers, as shown in - Table 1. Usually those patients may have a previous pathological history of: (1) pain episodes in the head, neck or shoulder girdle, (2) head or neck trauma, (3) inappropriate posture or postural habits, (4) bruxism for long periods, (5) manipulation of the teeth, jaw, or cervical spine, (6) cervical spine dysfunction and/or listhesis associated with the symptom. ${ }^{13,24}$

The following exclusion criteria were used: (1) previous experience with needles for therapeutic purposes, (2) formal contraindication to DN, such as chronic use of anticoagulants or the presence of hematologic diseases, (3) needle phobia or refusal of the proposed therapy, (4) use of medication for pain or tinnitus that could interfere in the study outcome, such as anti-inflammatory drugs and/or muscle relaxants, up

Table 1 Muscles suitable for placebo and therapeutic dry needling

\begin{tabular}{|l|}
\hline $\begin{array}{l}\text { Muscles suitable for placebo and therapeutic dry } \\
\text { needling }\end{array}$ \\
\hline Masseter \\
\hline Temporalis (medium fibers) \\
\hline Sternocleidomastoid \\
\hline Posterior Scalene \\
\hline Trapezius (descending fibers) \\
\hline Infraspinatus \\
\hline Splenius muscle of the head \\
\hline Medial and Lateral Pterygoids \\
\hline Rhomboid major \\
\hline Scapula Lift \\
\hline Digastric (posterior belly) \\
\hline
\end{tabular}


to 30 days prior to the initial assessment, (5) manual therapy for pain management up to 30 days before the initial assessment, and (6) patients with fibromyalgia.

All patients were evaluated by an otolaryngologist and underwent tonal, vocal and immittance audiometry, highfrequency audiometry, tinnitus psychoacoustic measurements, blood tests, and magnetic resonance imaging (MRI) in case of suspected retrocochlear lesion.

On the $1^{\text {st }}$ day of placebo DN, the selected subjects were submitted to: (1) physical reassessment for MTP, (2) measurement of degree of tinnitus discomfort and (3) cervical and/or shoulder girdle pain, both using VAS, (4) answering of the Tinnitus Handicap Inventory (THI) and of the Neck Disability Index - Brazil (NDI-BR) questionnaires, and (5) measurement of muscle tension using the Neutone (Try-All, Chiba City, Kanto, Japan) device. This device is capable of measuring muscle tension/muscle tone if the measurement condition does not change during follow-up and the position and posture of the patient are consistently replicated at every therapeutic session. ${ }^{19}$ Measurements placed perpendicular to the muscle to be tested by the Neutone device were established, performed in triplicate for each patient, always by the same researcher, in order to keep a uniform and constant pressure pattern as well as to prevent measurement misreading.

After collecting the measurement variables, the patient was instructed to lie down in ventral (prone) or dorsal (supine) decubitus, depending on the muscle suitable for treatment (see - Table $\mathbf{1}$ ).

\section{Placebo Dry Needling}

After proper positioning of the patient, the therapist made hand and local skin asepsis of the location to be needled with $70 \%$ alcohol and wore disposable latex gloves during the procedure. The marketed placebo kit (Asia-Med, Asia-Med Acupuncture Needle, Suhl, Turingia, Germany), had a guide and a needle (-Fig. 1). It was positioned over the selected MTP with the fixed guide for inserting the placebo needle into the skin. As soon as the needle guide was forced against the skin, it was retracted into the guide, which caused the patient to feel a tactile and/or visual illusion of true penetration (-Fig. 2). This procedure lasted for $\sim 1$ minute and the needle was then removed along with the guide. A very light
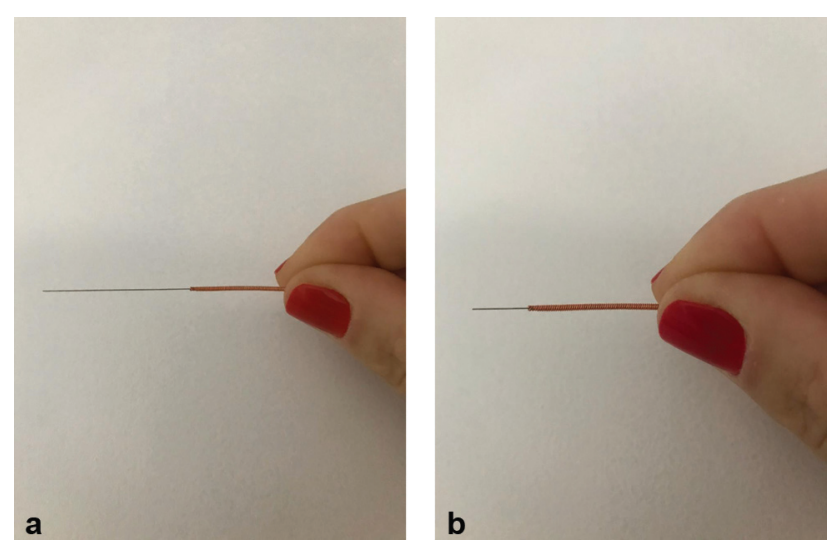

Fig. 1 Placebo needle.
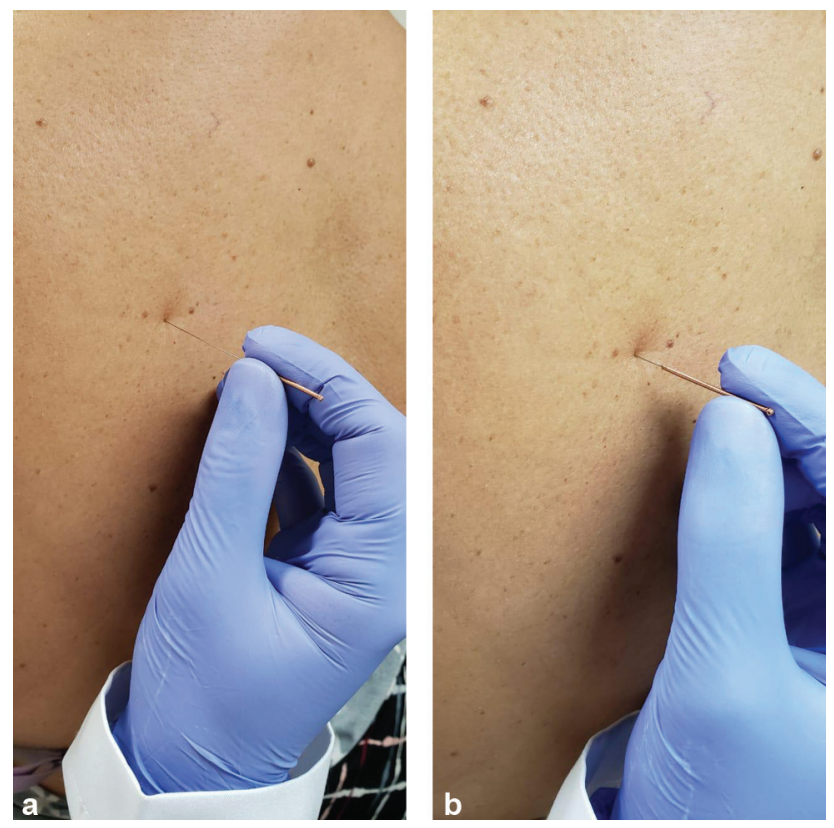

Fig. 2 Placebo needle: moments 1 and 2.

local pressure was then performed, followed by a nontherapeutic stretching of the "treated" muscle with free hands.

We are aware that, in physical therapy, one of the techniques used in rehabilitation is passive and therapeutic stretching of the muscle in question. In order to not interfere with the response to MTP treatment, in DN placebo, the muscular stretching was not fully performed, according to the technique protocol.

At the end of the procedure, the patient was instructed to sit again to have muscle tension measured using the Neutone device. At the end of this stage, the degree of tinnitus distress and pain was measured again using the VAS.

Dry needling placebo sessions were performed once a week by the same therapist for four consecutive weeks, and the THI and NDI-BR questionnaires were used again at the end of the $4^{\text {th }}$ placebo session. The measurements of the variables were done immediately after (at the end) the last session.

After an interval of 15 days (washout), all patients began the second phase of treatment, which was called therapeutic DN, with Asia-Med Acupuncture Needle CE 985270123 Suhl (-Fig. 3).

\section{Therapeutic Dry Needling}

After the washout period, the patient returned to the outpatient clinic, answered the THI and NDI-BR questionnaires, scored the tinnitus and pain discomfort through the VAS (before and after therapeutic DN), and had the measurements of muscle tension retraced by the Neutone device (before and after therapeutic DN). The needle, along with the guide, was held with the fingers. Subcutaneous skin penetration into the MTP was performed. The needle progressed $\sim$ between 1 and $2 \mathrm{~cm}$ away from the target MTP and was then angled at $30^{\circ}$. The DN protocol used in our study followed recommendations according to Dommerholt et al. ${ }^{25}$ 


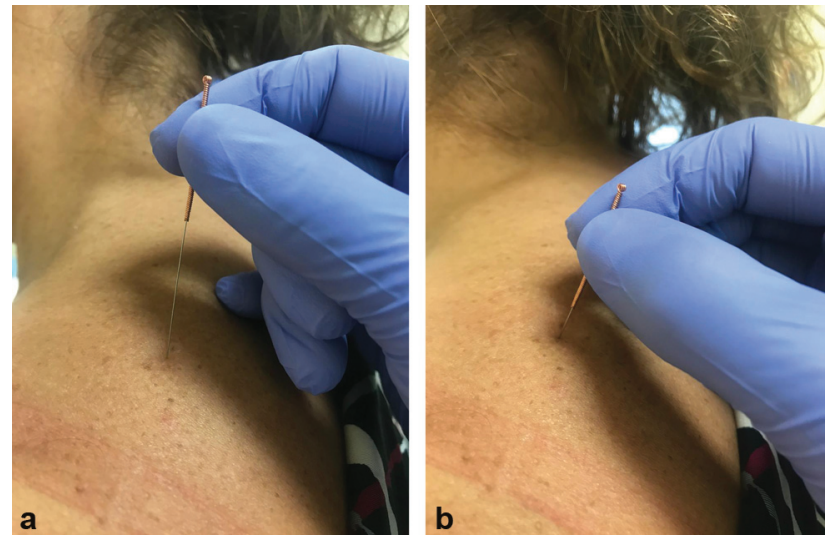

Fig. 3 Examples of therapeutic dry needling in the trapezius muscle (descending fibers).

The primary objective of the technique was to achieve a rapid contractile muscle response. ${ }^{25}$ This is an involuntary spinal reflex that contracts muscle fibers in the tense range, an effect that can be achieved both through digital pressure and through DN of the MTP. ${ }^{26}$ Long and thin needles with a diameter of $0.25 \times 30 \mathrm{~mm}$ were used for superficial body tissues, and needles with a diameter of $0.25 \times 40 \mathrm{~mm}$ were used for deeper body tissues.

Faced with a local twitch response (LTR), the needle remained in place for $\sim 30$ seconds to deplete the reflex and then, it was removed. The site was pressed again to check for reactions. In case of signal of nonabolished LTR, the needle was replaced, reinserted into the target MTP, and at this time held for up to 3 minutes. In some patients, intermittent needle twisting was required. ${ }^{27}$ The sessions took place once a week for a period of 4 consecutive weeks, and the THI and NDI-BR questionnaires were used again at the end of the $4^{\text {th }}$ therapeutic DN session (see Flowchart 1 for the timeline of the clinical trial). The measurements of the variables were done immediately after (at the end) the last session. We did not collect any more patient's data after that. Patients underwent to tinnitus treatment with other protocols.

\section{Statistical Analysis}

The statistical methodology included the Student t-test. The patient qualitative characteristics were described by means of their absolute and relative frequencies. Ages were described using their means and standard deviation (SD), median, minimum and maximum. ${ }^{28}$

The study variables are plotted in - Table $\mathbf{2}$, categorized according to the moment of the timeline in which they occurred, through their means and SDs, and their corresponding levels of statistical significance.

\section{Results}

Our sample comprised 16 patients with somatosensory chronic tinnitus who met the selection criteria and underwent placebo DN and therapeutic DN. None of the patients gave up treatment during the trial period. Among them, more than two thirds were female, with a mean age of
58 years old, with unilateral tinnitus in the majority of cases (82.2\%).

Comparing the two groups, we observed that the THI variable and its emotional domain reduced, in average, more in the therapeutic DN group than in placebo DN group, with a statistically significant difference $(p=0.024$ and $p=0.011$, respectively) and proven by the analysis of confidence intervals (CIs) for the means of change.

The tinnitus VAS for therapeutic DN showed improvement of the tinnitus discomfort, with a statistically significant mean difference, comparing the moments before and after therapy $(p<0.05)$. Conversely, the placebo DN showed no additional benefit in the treatment of tinnitus according to the study variables $(p>0.05)$.

There was a tendency to observe a statistically significant difference $(p=0.073)$ in the tinnitus VAS, since its reduction was greater after therapeutic DN than after placebo DN.

\section{Discussion}

\section{General Characteristics of the Sample}

One of the nonauditory comorbidities most commonly associated with tinnitus includes head and neck dysfunction (chronic spasms, muscle tension, neck pain, restriction of ROM), which usually precedes the onset of tinnitus. In a sample of 2,400 patients with tinnitus, 297 (12\%) reported that tinnitus started within 2 weeks after the trauma and, in most cases, $\sim 24$ hours after it. $^{29}$

The average age observed in our patients was 58 years old, and the ratio of tinnitus between females and males was 2:1. These findings are similar to those of the epidemiological field study conducted for the city of São Paulo. ${ }^{2}$ In this study, the symptom prevalence peak was at 65 years old. Out of 430 inhabitants with tinnitus (among 1,960 respondents), 273 (63.5\%) were female and 157 (36.5\%) were male. ${ }^{2}$ However, the aspect of gender may be controversial in the international literature, depending on the country and population studied.

A previous clinical experience with 150 patients examined between 1994 and 1996 at the Otorhinolaryngology Department of the Universidade de São Paulo reinforces that our local sample probably differs from that described as usual around the world, whose male individuals are the ones most frequently affected by the symptom. In this local study, the authors observed that $60 \%$ of the tinnitus patients were female and $40 \%$ were male. ${ }^{30}$ In addition, according to this same study, tinnitus was bilateral in $50 \%$ of the cases, unilateral in $39 \%$, and the others perceived it in the head. ${ }^{30}$ In our sample, unilateral tinnitus was found in $80 \%$ of the patients. This variation can be explained by the tinnitus subtype selected for the referred therapeutic intervention in our study.

A well-established concept in the literature is that one of the main characteristics of somatosensory tinnitus is that it occurs predominantly in one of the ears, ${ }^{24}$ mainly because such subtype of chronic tinnitus is generally associated with a somatic disorder of the head and neck, which affects primarily one side of the body. 


\section{FLOWCHART 1 (clinical trial timeline)}

\section{PATIENT WITH SOMATOSENSORY TINNITUS WITH ACTIVE OR LATENT MTP}

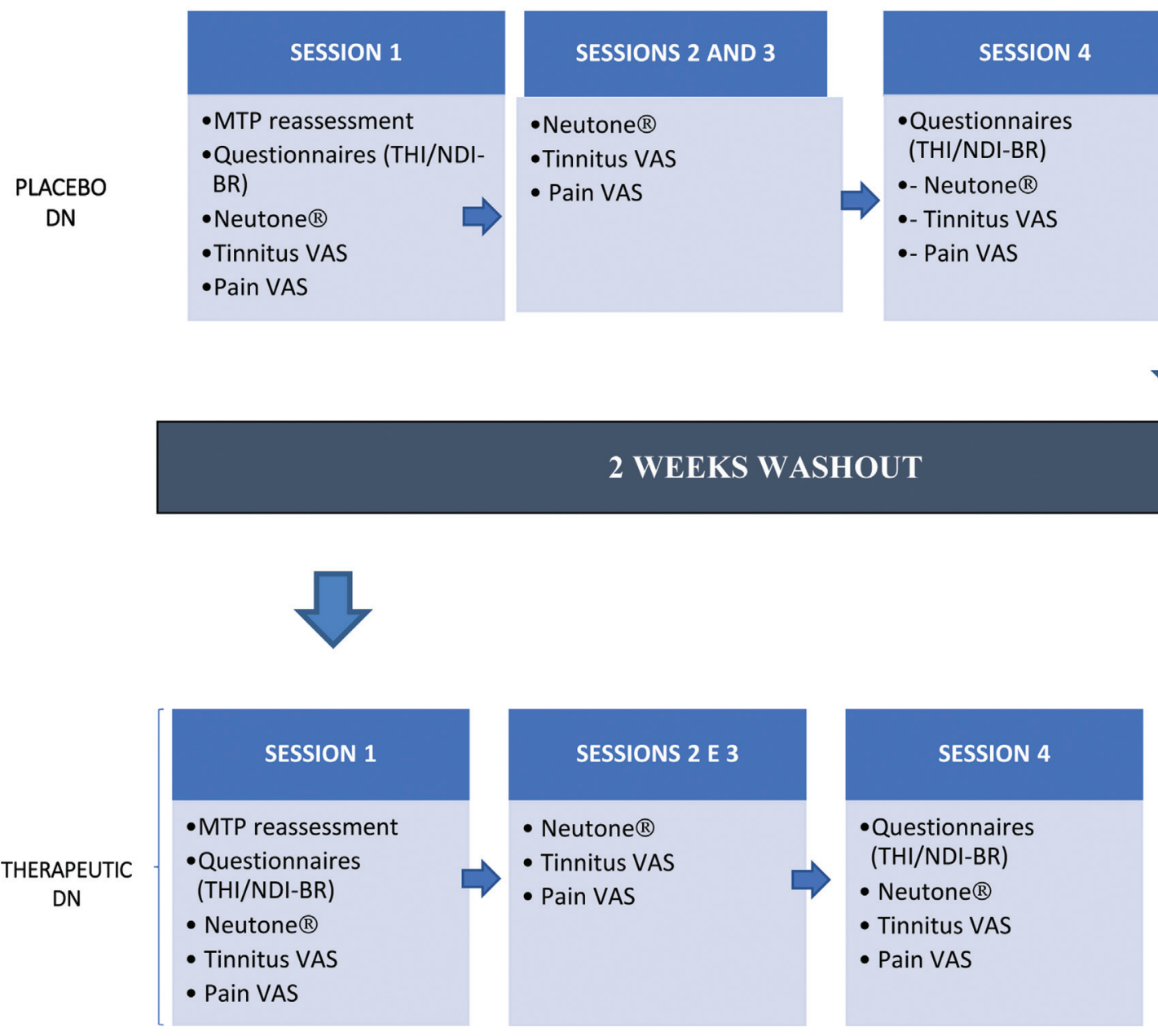

Flowchart 1 (clinical trial timeline).

\section{Measurement Variables}

Tinnitus Handicap Inventory Score Reduction when Comparing Placebo Dry Needling with Therapeutic Dry Needling

The THI is the only questionnaire validated and adapted to the Portuguese language up to the present time. ${ }^{31}$ It was created to quantify subjectively the impact of tinnitus on the quality of life of the patient and to classify reactions and discomfort into three main domains: (1) functional, (2) emotional, and (3) catastrophic. $^{32}$ The THI score of the same patient was tested at 2 different points in time to determine its replicability and reliability with a $95 \% \mathrm{CI}$ in the retest. In a previous study, to determine the minimum difference in the total THI score that could be considered clinically relevant, the absolute change in the questionnaire was compared with the Clinical Global Impression Improvement (CGI-I), which was the scale used to measure symptom severity, response, and treatment efficiency in 210 patients with tinnitus. ${ }^{33}$ A reduction of at least seven points could be considered to indicate a clinically relevant improvement in therapeutic clinical trials and to calculate the sample size. ${ }^{33}$

Our study revealed an improvement of $\geq 7$ points in the total THI score in $31.2 \%$ of the patients after placebo DN, and in $68.7 \%$ of the patients after therapeutic DN, considering a minimum change variation of 7 points, as mentioned above. ${ }^{33}$ The percentage of clinical improvement in patients submitted to placebo DN, although high, matches with the previous 


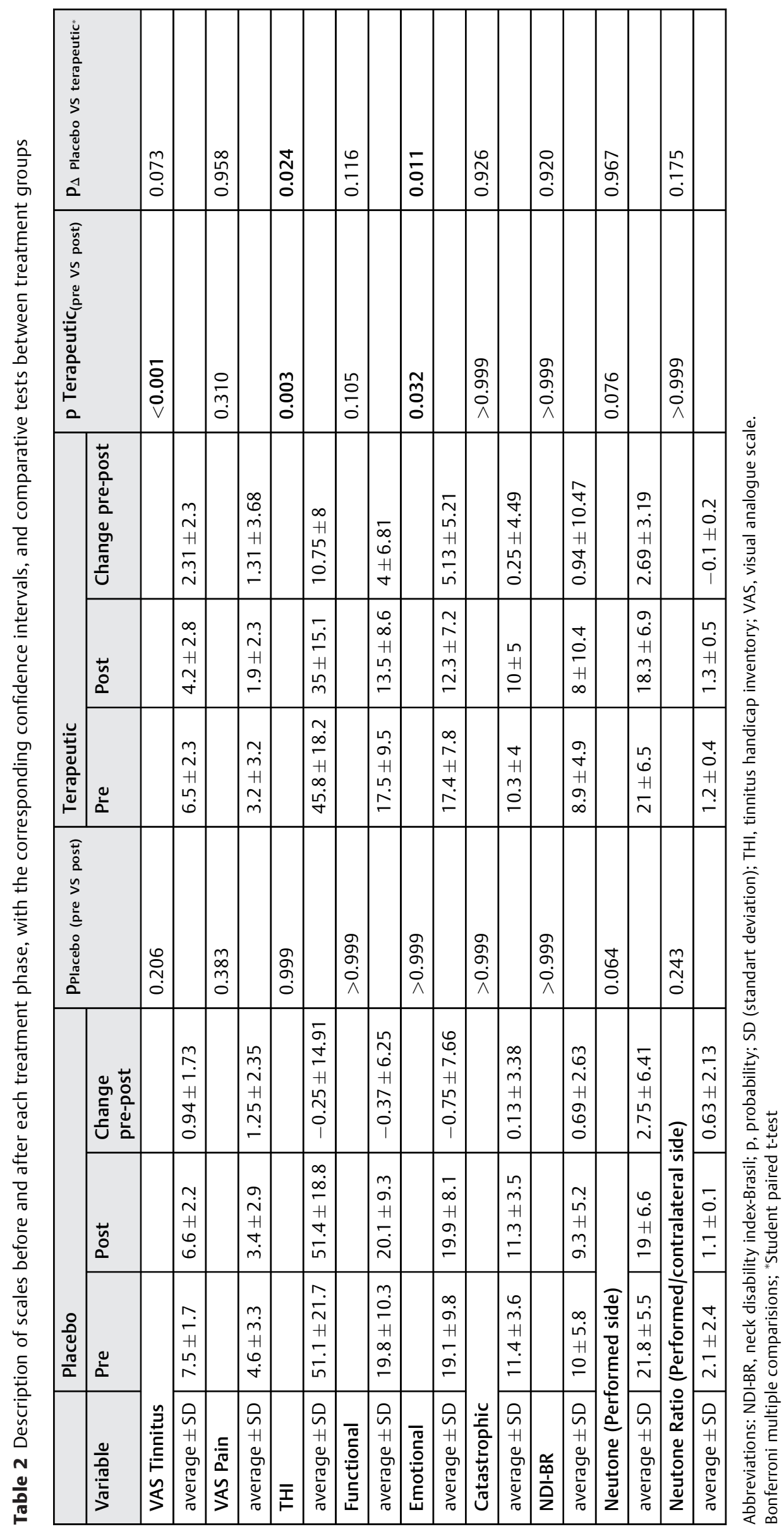


report in the literature, which indicates $40 \%$ improvement in chronic tinnitus patients due to a placebo effect. ${ }^{34}$

\section{Reduction of Emotional Domain Tinnitus Handicap Inventory Scores when Comparing Placebo Dry Needling with Therapeutic Dry Needling}

Our results also showed that DN interfered not only with the total THI score, but especially with its emotional domain, which is understandable, since our input sample included patients with average THI scores $>37$ points. Tinnitus severity is known to be directly correlated with stress, anxiety, depression, and emotional exhaustion levels. ${ }^{35}$ It is also known that, the higher the total score of the questionnaire, the higher the chances for this to happen, especially if the THI score is $\geq 38$ points. $^{36}$

Evidence suggests that structures involved in controlling the emotional neurophysiological and biological state and mood contribute directly to tinnitus modulation. ${ }^{35}$ In the brainstem, two structures have global influence on these functions, the cerulean locus (CL) and the dorsal raphe nucleus (DRN). ${ }^{35}$ The projections of these networks diffuse into the cerebral cortex and to tinnitus-generating sites through direct inputs to the cochlear nucleus and to the inferior colliculus. ${ }^{35}$ These $C L$ inputs use norepinephrine and serotonin as neuromodulators. ${ }^{35}$ The high prevalence of tinnitus whose psychoacoustic characteristics modulate through forceful contractions of head and neck muscles can, therefore, in some way reflect, in part, the synergy of brainstem connections between tinnitus-generating sites, somatosensory nuclei, trigeminal, cervical, mood and emotion modulating centers, including the $\mathrm{CL}$ and the DRN. ${ }^{35}$

One of the first brain functional imaging studies to provide evidence of the link between the limbic system and the central auditory cortex was performed by measuring cerebral blood flow in order to map regions responsive to tinnitus modulation. ${ }^{37}$ Patients whose voluntary orofacial movements modulated tinnitus intensity were compared with a control group and submitted to two different paradigms. The first paradigm was unilateral cochlear stimulation through pure tones, whose effect was the activation of the bilateral central auditory cortex in both groups (tinnitus and control). The fact that unilateral sound stimulation triggers a cortical effect in both cerebral hemispheres is fully understood, given the network and cross-neural connections decussation in the brainstem. In the second paradigm, there was modulation of tinnitus awareness through orofacial movements. Unexpectedly, in the latter, the change in cerebral blood flow was unilateral, which directly involves the central auditory pathways as being responsible for spontaneous neural activity that results in symptom modulation. Another relevant finding was that the sound stimulus activated a larger number of brain regions in the tinnitus group when compared with the control group. These findings, once again, support the neurophysiological model of Jastreboff ${ }^{1}$, which emphasizes the dramatic reorganization of the central auditory cortex and related and associated brain areas in tinnitus patients. Such wide range of brain areas is activated through neural connections of the central auditory cortex with the limbic system, through the hippocampus with the gateway to centers that mediate emotional control and memory. 37

It is also known that the acoustic environment can affect nonauditory brain regions as well. The amygdala and the hippocampus are the two largest areas of the limbic system that receive neural inputs directly or indirectly from the central auditory system, as well as the auditory thalamus (medial geniculate body). Therefore, acoustic stimuli can promote functional changes in the limbic system. In the same way, direct and indirect projections of the limbic system can also modulate neural activity in areas of the central auditory system. ${ }^{38}$

The response of the amygdala to sounds depends directly on their relevance in the sensory environment. Functional MRI in blind individuals, whose acoustic environment is more relevant than in individuals with normal visual acuity, shows a more intense amygdala response in the face of a sound stimulus with emotional connotation. Brain amygdala feedback to sensory stimuli occur even during sleep. However, the hippocampal-auditory system is essential for longterm hearing memory formation. The presence or absence of sound affects directly the structural and functional plasticity of the hippocampus. ${ }^{39}$

Experimental studies have shown that rats subjected to reversible conductive hearing loss developed depression, memory deficit, and reduction of dopamine, homovanilic acid and acetylcholinesterase activity. These behavioral and molecular changes disappeared after hearing rehabilitation. ${ }^{40}$

Neurocognitive tests have been used to compare skills between patients with moderate/severe/catastrophic tinnitus ( $\mathrm{THI} \geq 38$ ) with the normal population, matched by age, gender, and educational level. ${ }^{36}$ Results showed significantly higher deficits in the learning curve of tinnitus patients regarding evoked and associative memory and also when following commands and paying attention. ${ }^{36}$

In a systematic review article, clinical trials evaluating the impact of tinnitus on cognitive function were surveyed. Evidence suggests that the cortical cognitive impairment in patients with disabling chronic tinnitus possibly arises from a deficit in the processing and targeting of attention resources and in the conflict solving and executive control of responses. $^{41}$

Therefore, the reduction in the THI total scores and in its emotional domain seems to be supported by previous findings in literature showing a direct relationship among tinnitus, emotional state, cognition, and memory.

It seems that, in the moment we correct factors associated to tinnitus, such as MTP, and reduce the referred discomfort, we directly or indirectly help improve emotional and mood reactions related to the very awareness of tinnitus. Another possible explanation for the reduction in the emotional THI score would be the action of MTP deactivation in the serotoninergic system, which modulates various physiological and behavioral functions such as sleep, hunger, pain, mood, and emotions.

Most serotoninergic neurons are found in the DRN and in the medial raphe nucleus (MRN), with numerous projections 
on the auditory system, including the cochlear nucleus (CN), which centralizes most of them. Although the serotoninergic action in the auditory system is not well clarified, it is believed that it takes part in modulatory responses to simple and complex sounds, such as vocalization, for example. The dorsal cochlear nucleus (DCN) is a site of particular interest when studying the serotoninergic action in the auditory system, since it is the main terminal where thousands of multisensory inputs converge, which manages the location and orientation of the sound source and is an important center for tinnitus generation and modulation. ${ }^{42}$

It is well-known that an atypical serotonergic transmission underlies the pathogenesis of tinnitus, at one or more levels of the auditory pathways, previously documented by spontaneous hyperactivity found all over its network. ${ }^{43}$ This fact should contribute not only to the generation but also to the persistent tinnitus awareness in some patients, as well as to the emotional and mood reactions related to it. ${ }^{43}$

We can infer that the treatment of MTPs and their consequent improvement in our sample may have contributed to the regularization of this neural signaling pathway. The reduction in spontaneous hyperactivity of these multisensory connections could explain the reduction in the THI emotional domain score in these patients.

\section{Visual Analogue Scale of Tinnitus Discomfort before and after Therapeutic Dry Needling}

Clinical trials that study patients with subjective symptoms such as chronic pain depend on the adequate measurement of their disability as a requirement to verify the response to treatment. Although there is no gold standard method for measuring subjective symptoms, scales are adopted routinely in the clinical practice.

The VAS is a metric scale for intensity or frequency of symptoms that require direct objective measurement. The scale ranges from " $0 \mathrm{~cm}$ " which corresponds to "absence of the symptom" to " $10 \mathrm{~cm}$ " (unbearable symptom). The symptom score can be decoded as follows: mild (up to $3 \mathrm{~cm}$ ), moderate $(4$ to $7 \mathrm{~cm})$, and severe $(8$ to $10 \mathrm{~cm}$ ). The scale needs to be visually presented to the patient so that they can see it.

In our research, there was no statistically significant difference regarding the perception of tinnitus for treatments with placebo and therapeutic DN, as measured by the VAS, despite the tendency towards statistical deviation observed. We believe that this is because the sample size was smaller than initially designed. The screening for the inclusion and exclusion criteria made it very difficult for us to reach the desired sample size in a timely manner (a number of patients who could have been included in the research had had previous experiences with acupuncture). However, when analyzing exclusively the tinnitus before and after therapeutic DN, the variation in the VAS was statistically significant.

Currently, studies evaluating physical therapy treatments of somatosensory tinnitus are not comparable in terms of techniques used and of variables measured during the clinical follow-up of the patients. For this reason, we searched in the literature for clinical trials that used study variables such as the THI and the VAS. Among them, we have found transcutaneous electrical stimulation (TENS), manual therapy, occlusive treatments, laser, home cervical exercises, and muscle relaxation techniques for MTP.

The effect of TENS was tested in 65 patients with chronic tinnitus. ${ }^{44}$ The authors did not specify whether the patients had somatosensory tinnitus as a diagnosis or not. Cases with mild hearing loss and severe tinnitus were selected. The patients were treated twice a week for 4 consecutive weeks; 45 were treated with TENS and 20 with placebo stimulation. About $62 \%$ of the TENS-treated patients had tinnitus improvement, versus $10 \%$ of those in the placebo group. Although the improvement reported by patients was greater in the study group than in the placebo group, it was observed that the VAS for tinnitus discomfort decreased from 6.7 to 5.4 after TENS, and from 6.5 to 5.7 in the placebo group. In other words, there was no statistically significant difference between the two groups. The same occurred for the THI score, which decreased from 49.4 on average to 42.8 after TENS and rose from 44.5 on average to 45.2 in the placebo group.

In a recent clinical trial, interocclusal treatment was evaluated for a period of 3 years in 89 patients with tinnitus and mandibular muscle pain. ${ }^{45}$ The 64 patients who reached the end of the study had a mean reduction from 68.3 ( $100 \mathrm{~mm}$ scale) to 37.4 for tinnitus disability assessed by the VAS, after 1 year of trial. No significant reduction was observed in subsequent years. The number of affected painful muscles decreased from 7 to 2 in 1 year of therapy, and remained so for 2 consecutive years. The authors considered a change $\geq 20 \mathrm{~mm}$ in the VAS as clinically significant; $58 \%$ of the patients had this improvement within 1 year of therapy, and this was sustained in $43 \%$ of the cases for 2 consecutive years. About $46 \%$ of the patients reported a $50 \%$ reduction in the frequency and severity of tinnitus.

In a clinical trial, 20 patients with subjective tinnitus underwent 2 weekly sessions, up to a total of 12 complete sessions with continuous, low-intensity, red-wave $(630 \mathrm{~nm})$ and infrared $(808 \mathrm{~nm})$ diode laser, full dose of 120 Joules per ear and per session with the purpose of suppressing tinnitus temporarily. ${ }^{46}$ A paired t-test showed improvement in the VAS for tinnitus intensity from 5.7 before to 3.2 after completing therapy $(p<0.0001)$; the THI score reduced from 68.6 to 54.6 (14 points reduction on average).

Michiels et al. ${ }^{47}$ evaluated prognostic indicators for tinnitus disability reduction after cervical physiotherapy in patients with somatic cervicogenic tinnitus. Patients with moderate to severe tinnitus were assessed using the Tinnitus Functional Index (TFI) questionnaire and the cervical complaints questionnaire (Bournemouth Cervical Questionnaire $[\mathrm{QCB}]>14$ points). They received physical therapy treatment for the cervical spine, multimodal care, which included manual mobilization and home exercises of cervical selfmobilization for the flexor muscles. Twelve physical therapy sessions were held for 6 weeks. Measurement variables were documented before, immediately after treatment, and 6 weeks after the end of treatment. The patients whose tinnitus modulated (increased or decreased) simultaneously with cervical complaints had the lowest TFI scores, immediately after therapy $(p=0.001)$, and after late follow-up 
$(p=0.03)$. In addition, patients with low-frequency tinnitus and worsening after inadequate spinal postures were also responsible for lower TFI scores after treatment $(R=0.357)$, that is, they responded better to therapy.

We know that pain is a subjective symptom affected by several factors. In physical therapy, ways of treating discomfort are studied tirelessly in different groups and levels of severity. This would not be different for patients with somatosensory tinnitus. In our research, even though MTPs were present in the treated patients (as mentioned before), pain was not unbearable or incapacitating. For this reason, the VAS measurements for pain did not produce values statistically significant at the end of the trial. In the same way, the Neutone device, which assessed muscular tension and could be related to pain generated by MTPs, did not reveal statistically relevant changes.

Although these values were not significant, patients with somatosensory tinnitus searched for treatment for their main discomfort and were commonly not aware of the relationship between the somatic and auditory systems and that they may affect each other. When the patient is correctly diagnosed, we know there are greater chances for improvement of symptoms and, consequently, of their quality of life. ${ }^{13}$ Pain, which in many instances is not a simple clinical finding, becomes a great ally to stop tinnitus discomfort and fully improve it.

What could be observed when conducting the present research was the dedication of the patients, for they were never absent in the treatment sessions and believed in the improvement of their symptoms. And it really came true, for the efficacy of DN in the MTP of patients with chronic tinnitus was confirmed.

\section{Conclusion}

The therapeutic DN technique for MTPs in patients with chronic somatosensory tinnitus was effective in reducing the discomfort of the symptom measured by the THI (total score) and its emotional domain.

\section{Funding}

Coordenação de Aperfeiçoamento de Pessoal de Nível Superior Faculdade de Medicina da Universidade de São Paulo

\section{Conflict of Interests}

The authors have no conflict of interests to declare.

\section{References}

1 Jastreboff PJ. Phantom auditory perception (tinnitus): mechanisms of generation and perception. Neurosci Res 1990;8(04): 221-254

2 Oiticica J, Bittar RS. Tinnitus prevalence in the city of São Paulo. Braz J Otorhinolaryngol 2015;81(02):167-176

3 Levine RA. Somatic (craniocervical) tinnitus and the dorsal cochlear nucleus hypothesis. Am J Otolaryngol 1999;20(06): 351-362

4 Jousmäki V, Hari R. Parchment-skin illusion: sound-biased touch. Curr Biol 1998;8(06):R190
5 Dehmel S, Cui YL, Shore SE. Cross-modal interactions of auditory and somatic inputs in the brainstem and midbrain and their imbalance in tinnitus and deafness. Am J Audiol 2008;17(02): S193-S209

6 Manfredini D, Olivo M, Ferronato G, Marchese R, Martini A, Guarda-Nardini L. Prevalence of tinnitus in patients with different temporomandibular disorders symptoms. Int Tinnitus J 2015;19 (02):47-51

7 Bressi F, Casale M, Papalia R, et al. Cervical spine disorders and its association with tinnitus: The "triple" hypothesis. Med Hypotheses 2017;98:2-4

8 Travell JG, Simons DG, Simons LS. Travell \& Simons' myofascial pain and dysfunction: the trigger point manual. Second edition Philadelphia: Lippincott Williams \& Wilkins, 1 vol.

9 Yeng LT, Kaziyama HHS, Teixeira MJ. Myofascial painful syndrome. Rev Med 2001;80(n.spe1):94-110

10 Alvarez DJ, Rockwell PG. Trigger points: diagnosis and management. Am Fam Physician 2002;65(04):653-660

11 Travell JG, Simons LS, Simons DG. Dor e disfunção miofascial manual dos pontos-gatilho. 2 ed. Porto Alegre: Artmed; 2005

12 Ralli M, Salvi RJ, Greco A, et al. Characteristics of somatic tinnitus patients with and without hyperacusis. PLoS One 2017a12(11): 1-15

13 Sanchez TG, Rocha CB. Diagnosis and management of somatosensory tinnitus: review article. Clinics (São Paulo) 2011;66(06): 1089-1094

14 Akamatsu FE, Yendo TM, Rhode C, et al. Anatomical Basis of the Myofascial Trigger Points of the Gluteus Maximus Muscle. Biomed Res Int 2017;2017:4821968. Doi: 10.1155/2017/4821968

15 Simpson LL. Molecular pharmacology of botulinum toxin and tetanus toxin. Annu Rev Pharmacol Toxicol 1986;26:427-453

16 Imamura ST, Fischer AA, Imamura M, et al. Pain management using myofascial approach when other treatment failed. Phys Med Rehabil Clin N Am 1997;8(01):179-196

17 Björne A. Assessment of temporomandibular and cervical spine disorders in tinnitus patients. Prog Brain Res 2007;166:215-219. Doi: 10.1016/S0079-6123(07)66019-1

18 Herraiz C, Aparicio JM. [Diagnostic clues in pulsatile tinnitus (somatosounds)]. Acta Otorrinolaringol Esp 2007;58(09):426-433

19 Tachibana K, Ueki N, Uchida T, Koga H. Randomized Comparison of the Therapeutic Effect of Acupuncture, Massage, and Tachibana-Style-Method on Stiff Shoulders by Measuring Muscle Firmness, VAS, Pulse, and Blood Pressure. Evid Based Complement Alternat Med 2012;2012:989705. Doi: 10.1155/2012/989705

20 Wyant GM. Chronic pain syndromes and their treatment. II. Trigger points. Can Anaesth Soc J 1979;26(03):216-219. Doi: 10.1007/BF03006985

21 Amanda B, Manuela M, Antonia M, Claudio M, Gregorio B. Posturography measures and efficacy of different physical treatments in somatic tinnitus. Int Tinnitus J 2010;16(01):44-50

22 Michiels S, Naessens S, Van de Heining P, et al. The effect of physical therapy treatment in patients with subjective tinnitus: a systematic review. Front Neurosci 2016;10:545

23 Ong J, Claydon LS. The effect of dry needling for myofascial trigger points in the neck and shoulders: a systematic review and metaanalysis. J Bodyw Mov Ther 2014;18(03):390-398

24 Michiels S, Ganz Sanchez T, Oron Y, et al. Diagnostic Criteria for Somatosensory Tinnitus: A Delphi Process and Face-to-Face Meeting to Establish Consensus. Trends Hear 2018; 22:2331216518796403. Doi: 10.1177/2331216518796403

25 Dommertholt J, Fernandes-de-las-Peñas C. Trigger point dry needling: an evidenced and clinical-based approach. Churchill Livingstone: Elsevier; 2013:304

26 Hong CZ. Lidocaine injection versus dry needling to myofascial trigger point. The importance of the local twitch response. Am J Phys Med Rehabil 1994;73(04):256-263

27 Baldry P. Superficial versus deep dry needling. Acupunct Med 2002;20(2-3):78-81 
28 Kirkwood BR, Sterne JAC. Essential medical statistics. Cidade: Blackwell Science; 2006:502

29 Folmer RL, Griest SE. Chronic tinnitus resulting from head or neck injuries. Laryngoscope 2003;113(05):821-827

30 Sanchez TG, Bento RF, Miniti A, Camara J. Tinnitus: some characteristics and epidemiology. Clinical experience from Clinics Hospital of São Paulo University. Revista Bras Otorrino. 1997;63(03): 229-235

31 Paula Erika Alves F, Cunha F, Onishi ET, Branco-Barreiro FC, Ganança FF. [Tinnitus Handicap Inventory: cross-cultural adaptation to Brazilian Portuguese]. Pro Fono 2005;17(03):303-310

32 Newman CW, Sandridge SA, Jacobson GP. Psychometric adequacy of the Tinnitus Handicap Inventory (THI) for evaluating treatment outcome. J Am Acad Audiol 1998;9(02):153-160

33 Zeman F, Koller M, Figueiredo R, et al. Tinnitus handicap inventory for evaluating treatment effects: which changes are clinically relevant? Otolaryngol Head Neck Surg 2011;145(02):282-287

34 Duckert LG, Rees TS. Placebo effect in tinnitus management. Otolaryngol Head Neck Surg 1984;92(06):697-699

35 Theodoroff SM, Kaltenbach JA. The role of the brainstem in generating and modulating tinnitus. Am J Audiol 2019;28 (1S):225-238

36 Pierce KJ, Kallogjeri D, Piccirillo JF, Garcia KS, Nicklaus JE, Burton $\mathrm{H}$. Effects of severe bothersome tinnitus on cognitive function measured with standardized tests. J Clin Exp Neuropsychol 2012; 34(02):126-134

37 Simonetti P, Oiticica J. Tinnitus neural mechanisms and structural changes in the brain: the contribution of neuroimaging research. International Archives of Otorhinolaryngology 2015;19(03): 259-265

38 Kraus KS, Canlon B. Neuronal connectivity and interactions between the auditory and limbic systems. Effects of noise and tinnitus. Hear Res 2012;288(1-2):34-46
39 Klinge C, Röder B, Büchel C. Increased amygdala activation to emotional auditory stimuli in the blind. Brain 2010;133(Pt 6):1729-1736

40 Drago F, Nardo L, Rampello L, Raffaele R. Vestibular compensation in aged rats with unilateral labyrinthectomy treated with dopaminergic drugs. Pharmacol Res 1996;33(02):135-140

41 Tegg-Quinn S, Bennett RJ, Eikelboom RH, Baguley DM. The impact of tinnitus upon cognition in adults: A systematic review. Int J Audiol 2016;55(10):533-540

42 Tang Z-Q, Trussell LO. Serotonergic regulation of excitability of principal cells of the dorsal cochlear nucleus. J Neurosci 2015;35 (11):4540-4551

43 Thompson AM, Thompson GC, Britton BH. Serotoninergic innervation of stapedial and tensor tympani motoneurons. Brain Res 1998;787(01):175-178.

44 Lee SK, Chung H, Chung JH, Yeo SG, Park MS, Byun JY. Effectiveness of transcutaneous electrical stimulation for chronic tinnitus. Acta Otolaryngol 2014;134(02):159-167

45 Unell L, Ström D, Ekman K, Johansson A, Arnrup K, Carlsson GE. A 3-year study of patients with tinnitus and jaw muscle tenderness. Cranio 2019;37(05):304-309. Doi: 10.1080/08869634. 2018.1430097

46 Montazeri K, Mahmoudian S, Razaghi Z, Farhadi M. Alterations in auditory electrophysiological responses associated with temporary suppression of tinnitus induced by low-level laser therapy: a before-after case series. J Lasers Med Sci 2017;8(1, Suppl 1): S38-S45

47 Michiels S, Ganz SanchezT, Oron Y, Gilles A, Haider HF, Erlandsson S, Bechter K, Vielsmeier V, Biesinger E, Nam EC, Oiticica J, de Medeiros ÍRT, Bezerra RochaC, Langguth B, Van de Heyning P, De Hertogh W, Hall DA. Diagnostic criteria for somatosensory tinnitus: a Delphi processand face-to-face meeting to establish consensus. Trends Hear 2018;22:2331216518796403. Doi: 10.1177/2331216518796403 